\title{
GMR
}

\section{Conjunctiva reconstruction by induced differentiation of human amniotic epithelial cells}

\author{
S.P. Yang ${ }^{1}$, X.Z. Yang ${ }^{1}$ and G.P. $\mathrm{CaO}^{2}$ \\ 1'Department of Ophthalmology, People's Hospital of Taizhou City, Taizhou, China \\ 2Division of Liver Disease, People's Hospital of Taizhou, Jiangsu, China \\ Corresponding author: S.P. Yang \\ E-mail: yangshuiping929@163.com \\ Genet. Mol. Res. 14 (4): 13823-13834 (2015) \\ Received May 13, 2015 \\ Accepted August 21, 2015 \\ Published October 29, 2015 \\ DOI http://dx.doi.org/10.4238/2015.October.29.2
}

\begin{abstract}
In this study, we aimed to investigate the feasibility of directed differentiation of human amniotic epithelial cells into conjunctival epithelium under specific conditions as well as of constructing tissueengineered conjunctiva for ocular surface reconstruction. Human amniotic epithelial cells were cultured with induced denuded conjunctival matrix and conjunctival homogenate. Immunohistochemistry of cytokeratin-4, cytokeratin-13, and muc5ac as well as PAS staining were performed. The concentration of muc5ac at different times was measured using ELISA. The differentiated cells with quantum dots were transferred onto a denuded amniotic membrane to establish tissue-engineered conjunctiva and transplanted into a rabbit model with a conjunctival defect. After induction of human amniotic epithelial cells, differentiated cells showed conjunctival epithelium phenotype, while trace amounts of mu5ac in the culture medium measured by ELISA increased gradually within 1 to 7 days. Successfully tissue-engineered conjunctiva had similar structure as normal conjunctiva and was transplanted into a rabbit model with conjunctiva defect. After 2 weeks post-surgery, conjunctiva grafts
\end{abstract}


survived and were integrated. Immunohistochemistry showed conjunctival epithelium phenotype, positive cells were found in PAS staining. Thus, human amniotic epithelial cells could differentiate into conjunctival epithelium-like cells and goblet cells with partially physiological function, and we successfully restored ocular surface integrity in the rabbit model using tissue-engineered conjunctiva.

Key words: Amniotic epithelial cells; Trans-differentiation; Tissue engineering; Conjunctival reconstruction; Quantum dots; Denuded amniotic membrane

\section{INTRODUCTION}

The most common methods for treatment of conjunctival lesions caused by serious chemical injury, Stevens-Johnson syndrome, and ocular cicatricial pemphigoid include autograft/ allograft conjunctival transplants, buccal mucosa transplants, and amnion transplants (Stoiber and Grabner, 2005; Marchini et al., 2012; Ciralsky et al., 2013). Although autograft of conjunctiva and buccal mucosa transplantations is the ideal option, shortage of available tissue is a challenge. Postoperative rejection reaction is a major reason for failed autografts of conjunctiva and buccal mucosa. Repair of defective conjunctiva with amnion transplantation is preferred; however, two roadblocks exist at present: the presumption that adequate healthy conjunctival tissue is available, is invalid in case of serious conjunctival injury caused by burns or disease and postoperative amnion defects with regards to physiological function regardless of anatomic integrity posttransplantation, leading to discomfort or complications (Konomi et al., 2013; Ricciardelli et al., 2014). Transformation of conjunctival epithelial cells with secretary function after a small range of amnion transplantation to repair conjunctival damage due to burns has been recently reported (Eidet et al., 2012; Zhang et al., 2013). These encouraging results suggest that amniotic epithelial cells can differentiate into conjunctival epithelial cells under specific conditions. Therefore, successful duplication of conjunctival conditions in vitro to establish artificial conjunctiva in culture, with physiological function will be useful in treatment of refractory conjunctival diseases. In this study, we investigated the feasibility of bioengineered conjunctiva and complete reconstruction of the ocular surface by mimicking the directional differentiation of human amniotic epithelial stem cells into conjunctival epithelia in an in vitro microenvironment.

\section{MATERIAL AND METHODS}

\section{Materials}

Healthy, New Zealand rabbits (1.5 to $2.0 \mathrm{~kg}$ body weight, of either sex, $\mathrm{n}=16$ ) were purchased from the Medical Experimental Animal Centre of Guangdong Province, China.

Reagents used in the study included primarily, DMEM/F12 medium (Gibco, USA), fetal bovine serum (FBS; Hyclone, USA), trypsin (Hyclone, USA), type-Il collagenase (Gibco, USA), mucin 5 subtype AC (MUC5AC) ELISA kit (Uscn Life, USA), mouse anti-human CK4, CK13, and MUC5AC monoclonal antibodies (Fuzhou Maixin Biotech. Co., Ltd., Fuzhou Maxin Biotech Corp, China), cellular adhesive 200x (Beijing Applygen Biomart, China). 


\section{Differentiation of human amniotic epithelial cells}

\section{Culture of human amniotic epithelial cells}

Amniotic membrane from women who underwent caesarean section during delivery, who tested negative for hepatitis, syphilis, and HIV antibodies was obtained under sterile conditions, cut into small blocks of $1 \times 1 \mathrm{~mm}$, digested with $0.25 \%$ trypsin at $37^{\circ} \mathrm{C}$, blocked with $10 \% \mathrm{FBS}$, and centrifuged. The supernatant was digested with $0.25 \%$ type-Il collagenase at $37^{\circ} \mathrm{C}$, centrifuged, and incubated in DMEM medium containing 10\% FBS. When confluence was achieved, the cells were digested with trypsin and passaged.

\section{Decellularization of conjunctival matrix and preparation of conjunctival homogenate.}

The intact thin layer of conjunctiva was obtained from a freshly donated eyeball after corneal transplantation by clamping the bulbar conjunctiva with typing forceps, circular excision of the conjunctiva at the corneoscleral junction with corneal scissors, and blunt isolation along the conjunctival surface. The complete conjunctiva was digested with $0.25 \%$ Dispase II at $37^{\circ} \mathrm{C}$ for $2 \mathrm{~h}$ and $0.25 \%$ trypsin for $10 \mathrm{~min}$ to remove epithelia, conjugated with $1 \%$ glutaraldehyde at room temperature for $5 \mathrm{~min}$, digested with $0.25 \%$ DNAase at $37^{\circ} \mathrm{C}$ for $4 \mathrm{~h}$ and rinsed with phosphate buffered saline (PBS). The treated conjunctival matrix was flattened, HE stained, and observed under an inverted microscope as well as using an electronic microscope to confirm absence of residual epithelia. The conjunctiva was then cut into small pieces, homogenized, stored at $-40^{\circ} \mathrm{C}$ for $48 \mathrm{~h}$, followed by melting at room temperature (repeated 3 times), and centrifuged at $3500 \mathrm{r} / \mathrm{min}(1150 \mathrm{~g})$ for $30 \mathrm{~min}$. The supernatant was dialyzed at $4^{\circ} \mathrm{C}$ for $48 \mathrm{~h}$ and filtered through a $0.22-\mu \mathrm{m}$ filter. The dialysate $(10 \mathrm{~mL})$ was added to $90 \mathrm{~mL}$ DMEM medium containing $10 \%$ FBS to obtain $0.1 \mathrm{~g} / \mathrm{mL}$ conjunctival culture medium.

\section{Induced differentiation of human amniotic epithelia.}

The amniotic epithelia after 3 passaging were inoculated in decellularized conjunctival matrix at the density of $10^{5} \mathrm{cells} / \mathrm{mL}$. After cell adhesion, the resident cellular suspending solution was replaced with $0.1 \mathrm{~g} / \mathrm{mL}$ conjunctival culture medium. The cells were put in incubator for differentiation.

\section{Cytological identification}

\section{Immunohistochemical staining}

After differentiation for 5 days, the cells were incubated with mouse anti-human CK4, CK13, and MUC5AC as primary antibodies, and goat anti-mouse lgG for immunohistochemical staining. Fresh amniotic membrane was used as control. Positive controls provided in the staining kit were used as positive control and PBS was used to replace primary antibodies in the negative control.

\section{Periodic acid-Schiff (PAS) staining}

The amniotic epithelia, differentiated for 5 days and decellularized conjunctival matrix 
were fixed, embedded, sectioned, oxidized with $1 \%$ periodic acid, stained with Schiff solution, restained with hematoxylin, and mounted with neutral balsam.

\section{MUC5AC measurement using ELISA}

The differentiated cells were digested with $0.25 \%$ trypsin and centrifuged, and the supernatant was discarded. DMEM Medium containing 10\% FBS was added to the pellet and incubated at $37^{\circ} \mathrm{C}$ with $5 \% \mathrm{CO} 2$. After $1,3,5$, and 7 days of incubation, $0.8 \mathrm{~mL}$ culture medium was collected to measure the MUC5AC content according to the ELISA kit manual. The data were analyzed with curve expert 1.3 software to obtain the concentration.

\section{Engineered conjunctival membrane construction using decellularized conjunctiva as vector}

\section{Preparation of decellularized amniotic membrane}

Partial amniotic membrane was cut, digested with $0.25 \%$ trypsin at $37^{\circ} \mathrm{C}$ for $1 \mathrm{~h}$, rinsed with PBS, and flattened on a glass plate. Superficial epithelial cells on the glass plate were scraped off using a cellular scraper, leaving no residual cells as confirmed under an inverted microscope. The decellularized amniotic membrane was cut into $10 \times 10 \mathrm{~mm}$ blocks, smeared with cellular adhesive.

\section{Construction of engineered conjunctiva}

The differentiated cells at $1^{\text {st }}$ passage were incubated with quantum dot solution for $2 \mathrm{~h}$, followed by replacement with DMEM medium containing 10\% FBS and incubated for $24 \mathrm{~h}$. The absorption of quantum dots by differentiated cells was examined with a confocal microscope. These differentiated cells with quantum dots were inoculated in 6-well plates coated with decellularized amniotic membrane at a density of $10^{5}$ cells $/ \mathrm{mL}$. After adhesion to the amniotic membrane, the cells were transferred to transwell plates with addition of sufficient DMEM medium containing $10 \%$ FBS to ensure that the bottom of the decellularized amniotic membrane was in contact with the medium while the upper layer of cells were in contact with air. The air-liquid interface culture was incubated with a change of medium every alternate day.

\section{Transplantation of tissue-engineered conjunctiva}

\section{Animal model with conjunctival defect}

The right eyeball of 16 rabbits were transplanted with bulbar conjunctiva and fornical conjunctiva with a total size of $11 \times 13 \mathrm{~mm}$. Bleeding was stopped completely and the animals were divided into experimental group (tissue-engineered conjunctiva group) and control group (fresh amniotic membrane group), with 8 rabbits in each group. Then the tissue-engineered conjunctiva and fresh amniotic membrane with a size of $8 \times 10 \mathrm{~mm}$ were transplanted to the right eyes of rabbits with defective conjunctiva. There was a 2 to $3 \mathrm{~mm}$ area of defect between the normal conjunctiva and transplanted graft. 


\section{Postoperative examination}

The extent of atretoblepharia, integrity of graft, and surrounding inflammation were examined with slit-lamp microscopy after transplantation. The amniotic membrane and tissueengineering conjunctiva were removed 2 weeks later, for immunohistochemical staining, $\mathrm{HE}$ staining, PAS staining, and confocal microscope examination.

\section{Statistical analysis}

The data were analyzed using Statistical Product and Service Solutions (SPSS) 17.0 software for one-way ANOVA for multiple independent factors. $\mathrm{P}<0.05$ was set as significance level.

\section{RESULTS}

\section{Immunohistochemical examination of cells before and after differentiation}

The human amniotic epithelial cells did not express CK4, CK13, and MUC5AC prior to differentiation. However, the cells were found to express CK4, CK13, and MUC5AC post differentiation, demonstrated by the appearance of brown granules in cytoplasm (Figure 1).

\section{PAS staining}

PAS staining showed sporadic purple differentiated cells in paraffin-embedded sections (Figure 2).

\section{Measurement of MUC5AC with ELISA}

Analysis using professional software, curve expert 1.3 revealed that the concentration of MUC5AC at $1,3,5$, and 7 days was $1.757 \pm 0.883,2.397 \pm 0.752,2.816 \pm 1.088$, and 3.543 $\pm 1.001 \mu \mathrm{g} / \mathrm{L}$, respectively. One-way ANOVA with SPSS 13.0 indicated that there was significant difference every 2 days $(P<0.05)$.

\section{Tissue-engineered conjunctiva}

Quantum dots adhered to differentiated cells at $2 \mathrm{~h}$ of culture, displaying an even, pink color distribution (Figure 3A). At 7 days, decellularized amniotic membrane showed 2 to 3 layers of cells, which increased to 4 to 5 layers at 14 days. The cells were closely linked to basal membrane, forming a epithelia-basal membrane structure similar to normal rabbit conjunctiva. At 7 and 14 days, confocal microscope scans indicated that the differentiated cells labeled by quantum dots showed complete and regular morphology, were well-organized and compact, and had no obvious decay of fluorescent density (Figure $3 \mathrm{~B}, \mathrm{C}$ ).

\section{Postoperative examination}

Slit-lamp microscope examination showed that grafts in the 2 groups were in place and 
complete with mild edema within the graft and surrounding conjunctiva. The edema disappeared several days later. The conjunctival epithelia migrated toward the graft after 10 days. There was no atretoblepharia observed in the 2 groups.

HE staining showed that the engineered conjunctiva was covered by 2 to 5 layers of cells with slight infiltration of inflammatory cells in the deep layer while the amniotic membrane in the control group was covered by only a single layer of epithelia (Figure $4 \mathrm{~A}, \mathrm{~B}$ ).
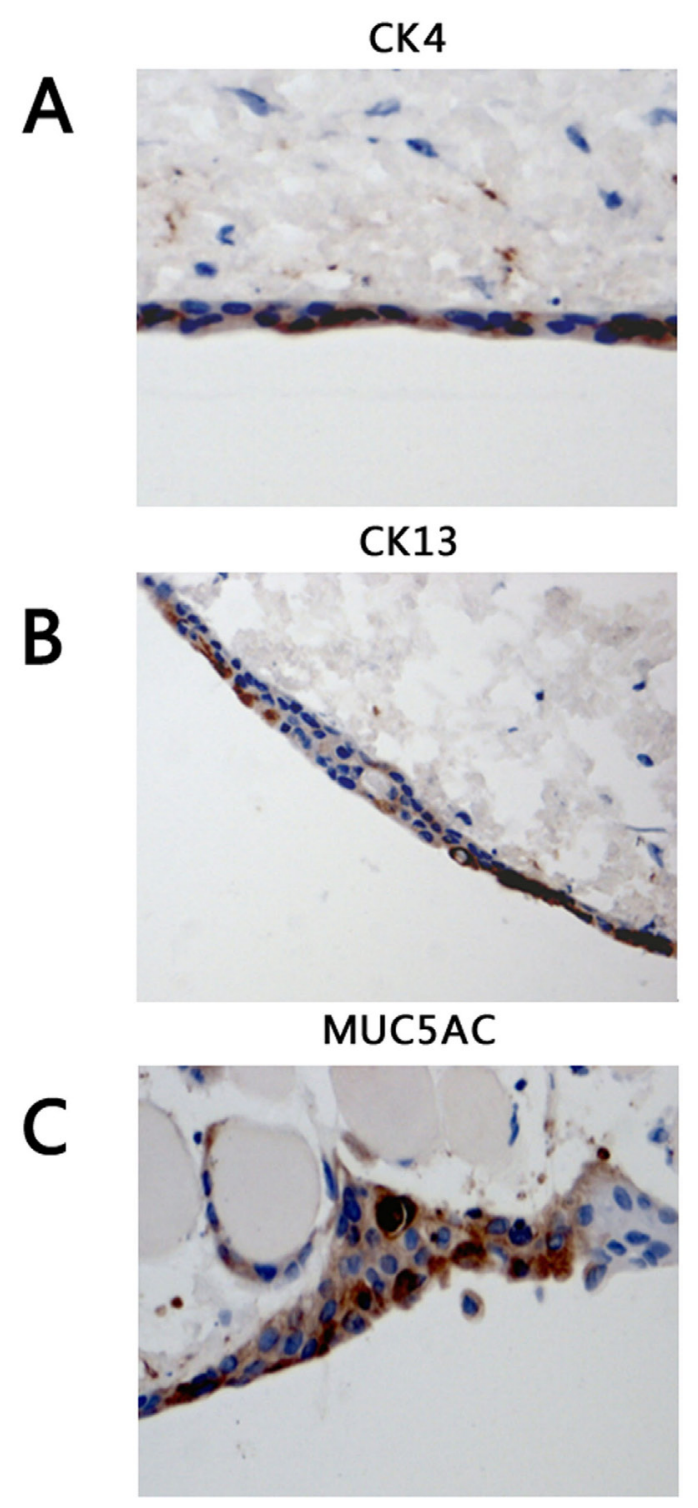

Figure 1. A. Expression of CK4 in human amniotic epithelia after differentiation. B. CK13 in human amniotic epithelia after differentiation. C. MUC5AC in human amniotic epithelia after differentiation. 


\section{Differentiated cells}

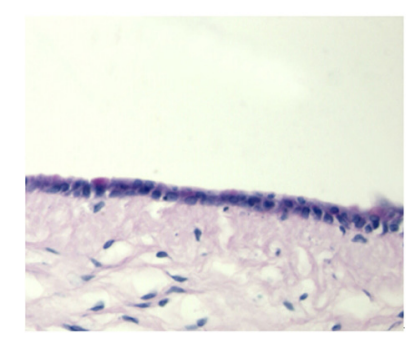

Figure 2. Sporadic purple differentiated cells with positive PAS.

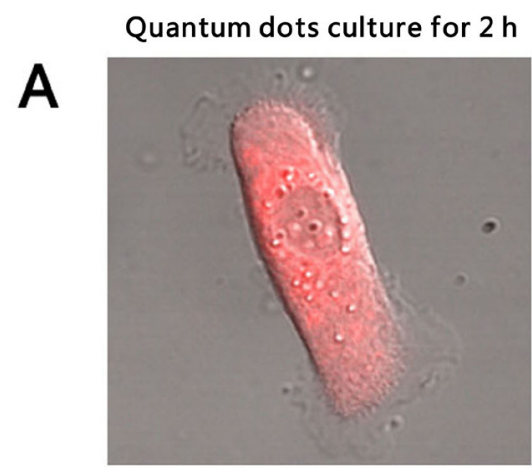

Multiple layers of cells

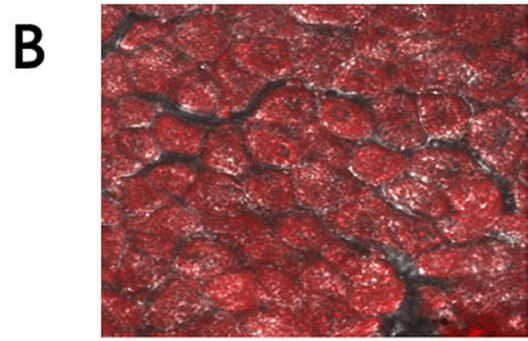

Multiple layers of cells

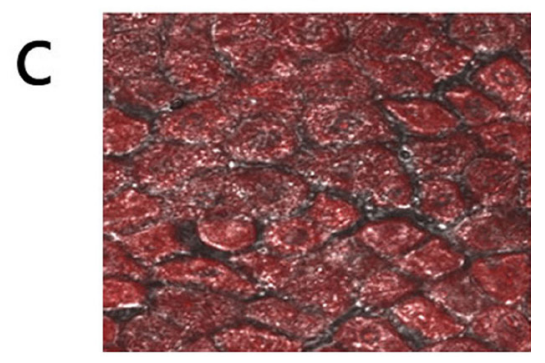

Figure 3. A. Quantum dot culture for $2 \mathrm{~h}$ showing pink staining in the membrane, cytoplasm and nuclei. B. Confocal microscope image of engineered conjunctiva at 7 days showing multiple layers of cells in deep red, clear cellular structure, complete and regular morphology, and well-organized and compact structure. C. Confocal microscope image of engineered conjunctiva at 14 days showing multiple layers of cells in deep red, clear cellular structure, complete and regular morphology, and well-organized and compact structure. 

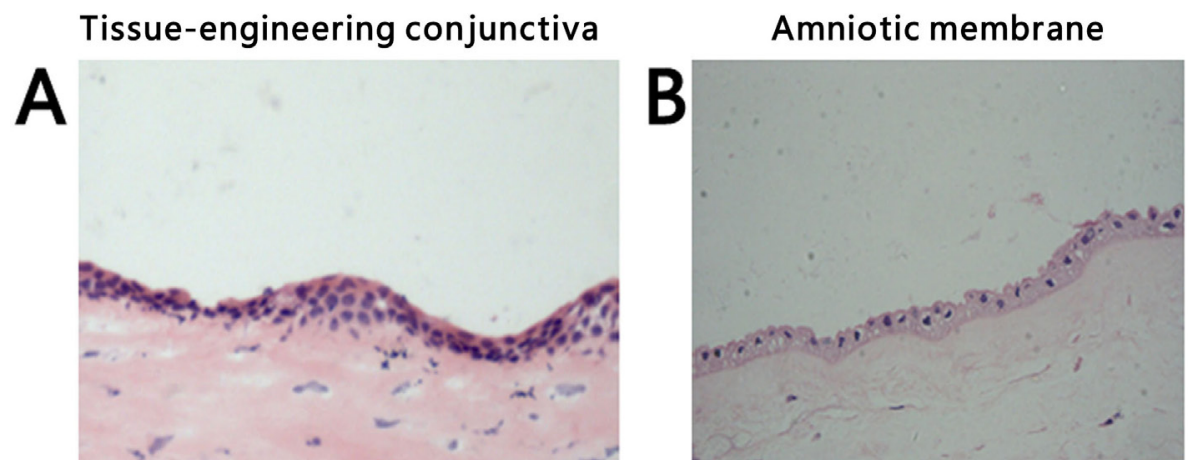

Figure 4. HE staining in tissue-engineering conjunctiva (A) HE staining in amniotic membrane (B).

Immunohistochemical staining showed that CK4, CK13, and MUC5AC were expressed in engineered conjunctiva but not in the amniotic membrane at day 14 after transplantation (Figure 5).

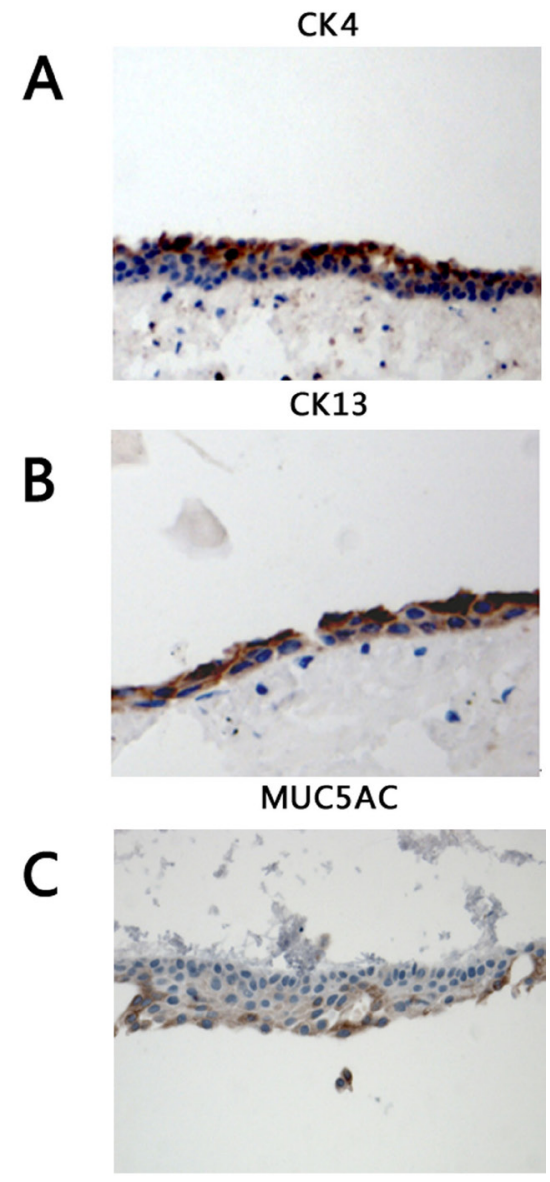

Figure 5. A. Expression of CK4 in tissue-engineering conjunctiva. B. CK13 in engineered conjunctiva. C. MUC5AC in engineered conjunctiva. 
PAS staining showed that there were sporadic purple positive cells in the cell layers of engineered conjunctiva graft (Figure 6).

\section{Tissue-engineering conjunctiva}

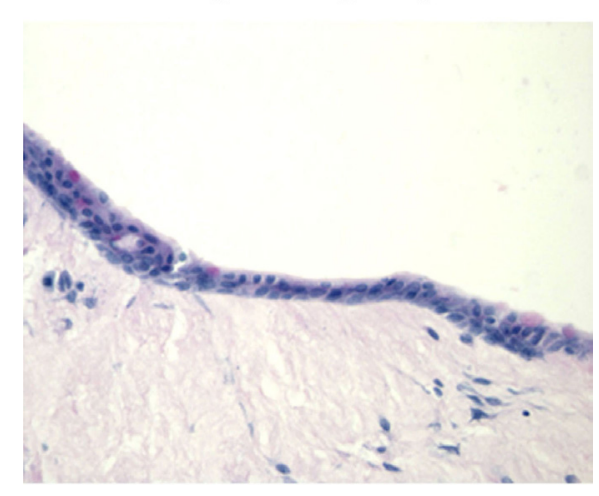

Figure 6. Positive PAS cells in engineered conjunctiva.

Images using confocal microscope indicated that the cells in engineered conjunctiva were well-organized with regular morphology and showed an evenly distributed, deep-red fluorescence (Figure 7).

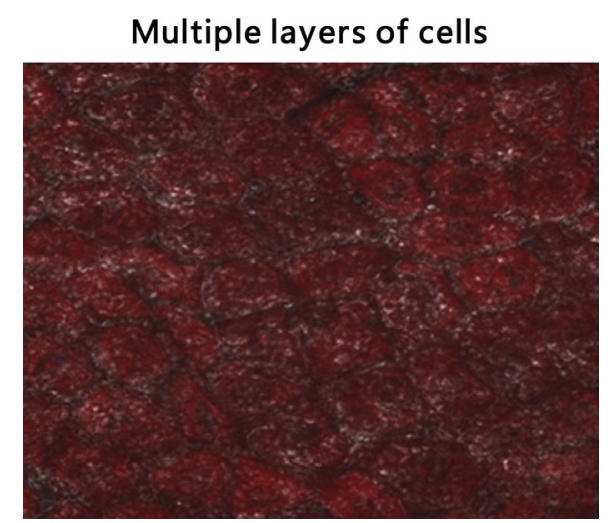

Figure 7. Magnified confocal images of cells within engineered conjunctiva in deep-red color, showing complete and regular morphology, and well-organized structure.

\section{Discussion}

\section{Directional differentiation induced in human amniotic membrane}

Stem cells and their directional differentiation are the hotspot of current study. So far, it is acknowledged that the microenvironment (niche) plays an important role in directional differentiation of stem cells and determines the fate of stem cells (Espana et al., 2003; Dua et al., 2005). The niche facilitates the modulation of stem cells through mutual spatiotemporal dialog with stem cells (Moore and Lemischka, 2006). 
Human amniotic epithelia are adult stem cells, with multiple differentiation potentials, similar to embryonic stem cells (Miki et al., 2005), are advantageous due to properties such as low immunogenicity, absence of oncogenicity, high availability, and absence of ethical concerns regarding their use. Therefore, human amniotic epithelia are the ideal choice as seeding cells in bio-tissue engineering and have been reported to differentiate into multiple tissues and cells (Fliniaux et al., 2004; Insausti et al., 2014; Zeng et al., 2014). In this study, we used human amniotic epithelia as seeding cells to investigate the directional differentiation of stem cells into fornical conjunctiva while mimicking the in vivo microenvironment using conjunctival homogenate and decellularized conjunctival matrix. After incubation in this microenvironment, the seeding cells expressed specific epithelial antigens such as CK4, CK13, and MUC5AC, demonstrated by immunohistochemical staining, as purple positive cells by PAS staining and markedly increased micro-secretion of MUC5AC measured using ELISA, and expressed MUC5AC gene, as confirmed by RT-PCR. These results suggested that conjunctival homogenate and decellularized conjunctival matrix can be used as in vitro microenvironment to induce directional differentiation of stem cells. Under this induction conditions, human amniotic epithelia have been successfully differentiated into cells with the phenotype of conjunctival epithelia and goblet cells with similar secretory function as normal conjunctival epithelia [ref], which further implies that the engineered conjunctiva displayed physiological functions similar to normal conjunctiva and has potential therapeutic applications.

\section{Quantum dot labeling to trace differentiated cells}

Quantum dots (QDs) are semiconductor nanocrystals composed of II-VI or III-VI family elements. QDs are widely used in biological studies as novel, non-isotopic, and highly sensitive fluorescence-labeled probes (Walker et al., 2011; Luo et al., 2014). For example, Ohyabu et al. clearly demonstrated the dynamic imaging procedure by labeling mesenchymal stem cells with QDs (Ohyabu et al., 2009). In this study, we labeled live cells with QDs and clearly observed labeling of cells within $2 \mathrm{~h}$, which showed good physiological conditions during the 2 weeks of tissue-engineering procedure. This result suggested that QDs have properties of low cytotoxicity, stable fluorescence, good biological compatibility, and are an ideal tracing marker for live samples, a promising combination for future studies.

\section{Tissue-engineering conjunctiva in re-constructing complete eye surface of model rabbits}

Simple amniotic membrane transplantation requires cells of surrounding healthy conjunctiva to move out and cover the surface of the amniotic membrane in order to achieve re-construction of the eye surface (Xu et al., 2012). For serious defects of the conjunctiva, simple amniotic membrane transplantation is challenging. It has been reported that successfully transplanted human amniotic membrane can transform into conjunctival epithelia-like cells and that the surrounding conjunctival tissue is sufficient to create the microenvironment required for directional differentiation of amniotic epithelia (Giasson et al., 2006; Wang et al., 2006; Fatima et al., 2008). However, diseases involving serious conjunctival defects such as serious conjunctiva burn, Stevens-Johnson syndrome, and ocular pemphigoid lack the microenvironment required for differentiation of amniotic membrane, which results in final dissolution and abscission of the amniotic membrane (Giasson et al., 2006; Wang et al., 2006; Fatima et al., 2008). 
The amniotic membrane has similar histological components as the basal membrane of ocular superficial epithelium and can secret cytokines such as alkaline fibroblastic growth factor and epidermal growth factor to promote epithelial adhesion, migration, and induced differentiation (Lee et al., 2006; Deolinda et al., 2007; Gicquel et al., 2009; Wolbank et al., 2009), and is widely used in the ophthalmology clinic. Owing to the removal of epithelia, the immunogenicity and rejection reaction are further decreased; therefore, the decellularized amniotic membrane is an ideal vector to construct engineered transplantation material for the surface of the eye (Koizumi et al., 2007). In this study, we constructed engineered conjunctiva using decellularized amniotic membrane as vector and human amniotic epithelia as seeding cells to repair the defective conjunctiva in an animal model. There was no postoperative atretoblepharia observed and the graft was in place at the end of the study. The conjunctival epithelia migrated toward the graft after 10 days. Immunohistochemical staining confirmed that the differentiated cells displayed epithelial phenotype of the conjunctiva. HE staining showed that the engineered conjunctiva had 2 to 5 layers of closely aligned cells. Confocal imaging indicated that the differentiated cells labeled by QDs were in regular morphology and in alignment. These results suggested that the transplanted cells survived well and adapted to the microenvironment of the live conjunctival sac and that the engineered conjunctiva showed good tissue compatibility in the animal model to allow complete reconstruction of the eye surface.

\section{Problems and prospects}

The results from this study preliminarily indicate the feasibility of constructing engineered conjunctiva and reconstruction of the eye surface using human amniotic epithelia as a viable option for seeding cells. However, several challenges remain: the detailed mechanism of differentiation induced by the niche, the method of immortalization of differentiated cells, the physiological functions of tissue-engineering conjunctiva, etc. are still unclear. Using multidisciplinary techniques of tissue-engineering, biomaterials, and ophthalmology, the reconstruction of eye surface using engineered tissue will be an effective approach for treatment of refractory ocular surface disease.

\section{Conflicts of interest}

The authors declare no conflict of interest.

\section{References}

Ciralsky JB, Sippel KC and Gregory DG (2013). Current ophthalmologic treatment strategies for acute and chronic StevensJohnson syndrome and toxic epidermal necrolysis. Curr. Opin. Ophthalmol. 24: 321-328.

Deolinda de Oliveira Pena J, Melo GB, Gomes JA, Haapalainen EF, et al. (2007). Ultrastructural and growth factor analysis of amniotic membrane preserved by different methods for ocular surgery. Arq. Bras. Oftalmol. 70: 756-762.

Dua HS, Shanmuganathan VA, Powell-Richards AO, Tighe PJ, et al. (2005). Limbal epithelial crypts: a novel anatomical structure and a putative limbal stem cell niche. Brit. J. Ophthalmol. 89: 529-532.

Eidet JR, Utheim OA, Raeder S, Dartt DA, et al. (2012). Effects of serum-free storage on morphology, phenotype, and viability of ex vivo cultured human conjunctival epithelium. Exp. Eye Res. 94: 109-116.

Espana EM, Kawakita T, Romano A, Di Pascuale M, et al. (2003). Stromal niche controls the plasticity of limbal and corneal epithelial differentiation in a rabbit model of recombined tissue. Invest. Ophthalmol. Vis. Sci. 44: 5130-5135.

Fatima A, Iftekhar G, Sangwan VS and Vemuganti GK (2008). Ocular surface changes in limbal stem cell deficiency caused by chemical injury: a histologic study of excised pannus from recipients of cultured corneal epithelium. Eye 22: 1161-1167.

Fliniaux I, Viallet JP, Dhouailly D and Jahoda CA (2004). Transformation of amnion epithelium into skin and hair follicles. Differentiation 72: 558-565. 
Giasson CJ, Bouchard C, Boisjoly H and Germain L (2006). Amnion and ocular surface problems. Med Sci 22: 639-644.

Gicquel JJ, Dua HS, Brodie A, Mohammed I, et al. (2009). Epidermal growth factor variations in amniotic membrane used for ex vivo tissue constructs. Tissue Eng. 15: 1919-1927.

Insausti CL, Blanquer M, Garcia-Hernandez AM and Castellanos G (2014). Amniotic membrane-derived stem cells: immunomodulatory properties and potential clinical application. Stem Cells Clon. 7: 53-63.

Koizumi N, Rigby H, Fullwood NJ, Kawasaki S, et al. (2007). Comparison of intact and denuded amniotic membrane as a substrate for cell-suspension culture of human limbal epithelial cells. Graefes Arch. Clin. Exp. Ophthalmol. 245: 123-134.

Konomi K, Satake Y, Shimmura S, Tsubota K, et al. (2013). Long-term results of amniotic membrane transplantation for partial limbal deficiency. Cornea 32: 1110-1115.

Lee JH, Ryu IH, Kim EK, Lee JE, et al. (2006). Induced expression of insulin-like growth factor-1 by amniotic membraneconditioned medium in cultured human corneal epithelial cells. Invest. Ophthalmol. Vis. Sci. 47: 864-872.

Luo L, Liu Z, Li J and Zhu J (2014). Fluorescence resonance energy transfer based immunosensing of human IgG by using quantum dot/ GlgG-gold nanoparticles/ IgG conjugation. J. Nanosci. Nanotechnol. 14: 4036-4042.

Marchini G, Pedrotti E, Pedrotti M, Barbaro V, et al. (2012). Long-term effectiveness of autologous cultured limbal stem cell grafts in patients with limbal stem cell deficiency due to chemical burns. Clin. Experiment. Ophthalmol. 40: 255-267.

Miki T, Lehmann T, Cai H, Stolz DB, et al. (2005). Stem cell characteristics of amniotic epithelial cells. Stem Cells 23: 15491559.

Moore KA and Lemischka IR (2006). Stem cells and their niches. Science 311: 1880-1885.

Ohyabu Y, Kaul Z, Yoshioka T, Inoue K, et al. (2009). Stable and nondisruptive in vitrol in vivo labeling of mesenchymal stem cells by internalizing quantum dots. Hum. Gene Ther. 20: 217-224.

Ricciardelli G, Ceccuzzi R, Raneri M, Scalisi A, et al. (2014). Management of recurrent corneal ulcers: use of amniotic membrane. Eur. J. Ophthalmol. 24: 793-796.

Stoiber J and Grabner G (2005). Clinical management of severe ocular surface disease. Klin. Mbl. Augenheilk. 222: 533-551.

Walker KA, Doak SH and Dunstan PR (2011). Mechanisms of cell-cell adhesion identified by immunofluorescent labelling with quantum dots: a scanning near-field optical microscopy approach. Ultramicroscopy 111: 1200-1205.

Wang M, Yoshida A, Kawashima H, Ishizaki M, et al. (2006). Immunogenicity and antigenicity of allogeneic amniotic epithelial transplants grafted to the cornea, conjunctiva, and anterior chamber. Investig. Ophthalmol. Vis. Sci. 47: 1522-1532.

Wolbank S, Hildner F, Redl H, van Griensven M, et al. (2009). Impact of human amniotic membrane preparation on release of angiogenic factors. J. Tissue Eng. Regen. Med. 3: 651-654.

Xu B, Fan TJ, Zhao J, Sun A, et al. (2012). Transplantation of tissue-engineered human corneal epithelium in limbal stem cell deficiency rabbit models. Int. J. Ophthalmol. 5: 424-429.

Zeng W, Li Y, Zeng G, Yang B, et al. (2014). Transplantation with cultured stem cells derived from the human amniotic membrane for corneal alkali burns: an experimental study. Ann. Clin. Lab. Sci. 44: 73-81.

Zhang ZD, Ma HX, Chen D, Li M, et al. (2013). A novel technique of modified continuous blanket suture for amniotic membrane fixation in severe ocular surface diseases. JAMA Ophthalmol. 131: 941-947. 